\title{
Urological Management of Extramural Malignant Ureteric Obstruction: A Survey of Irish Urologists
}

\author{
Eabhann M. O'Connor Gregory J. Nason \\ Department of Urology, Cork University Hospital, Wilton, Ireland
}

\author{
Eamon A. Kiely
}

\section{Key Words}

Obstructive uropathy • Ureteral stent

\begin{abstract}
Introduction: The absence of guidelines in the management of extramural malignant ureteric obstruction leads to confusion in decision making and in the interaction between urology and other clinical disciplines. In this study, we surveyed consultant urologists with the goal of achieving a better consensus on optimal management options. Methods: A multiple choice survey was sent via the online survey tool "SurveyMonkey" to all consultant urologists practicing in the Republic of Ireland. Results: There was a response rate of $57.5 \%$ ( $n=23$ ). Twenty-two $(96 \%)$ consultants consider the use of percutaneous nephrostomy with placement of antegrade ureteric stent but only $22 \%(n=5)$ would consider using a metallic stent. Eleven (48\%) respondents favor retrograde stenting in the first instance with an equal proportion choosing an antegrade method. Nine (39\%) consultants perform the initial stent change at 4-6 months, 8 (35\%) at 2-4 months, and 1 at $<2$ months and 6-10 months respectively. Total $59 \%(n=13)$ of respondents felt that the duration of expected patient survival influenced their decision and agreement to stent with $42 \%(n=8)$ saying this survival would need to be $>6$ months and $82 \%(n=18)$ were generally happy with the level of ongoing communication between urology and the primary service managing the patient. Conclusion: There is a lack of consensus regarding the management of this challenging problem, particularly with
\end{abstract}

\section{KARGER}

(C) 2017 S. Karger AG, Basel

Fax +4161306 1234

E-Mail karger@karger.com Accessible online at:

www.karger.com www.karger.com/cur regard to timing of first stent change and whether to initially use an antegrade or retrograde approach. This reflects the heterogeneous patient cohort and the important factors of life expectancy and patient co-morbidities.

Copyright @ 2017 S. Karger AG, Basel

\section{Introduction}

Extramural ureteric obstruction caused by advanced urological or non-urological malignancy is a challenging and heterogenous clinical entity for urologists [1]. Presenting symptoms are often varied and can range from vague and nonspecific symptoms such as flank discomfort or lethargy, to those of the intense pain, nausea and vomiting associated with acute obstruction [2]. The diverse patient cohort is also onerous, with age, site of primary malignancy, pre-existing medical co-morbidities and primary physician/patient wishes all important variables $[1,3,4]$.

The therapeutic goal of urinary drainage in malignant disease is to provide symptomatic relief of ureteric obstruction while preserving renal function and optimizing quality of life $[1,5]$. Management strategies include percutaneous nephrostomy tube placement with or without antegrade stent insertion, retrograde stent placement or use of metallic stents $[6,7]$. Although the reported health-related quality of life for patients with a percuta- 


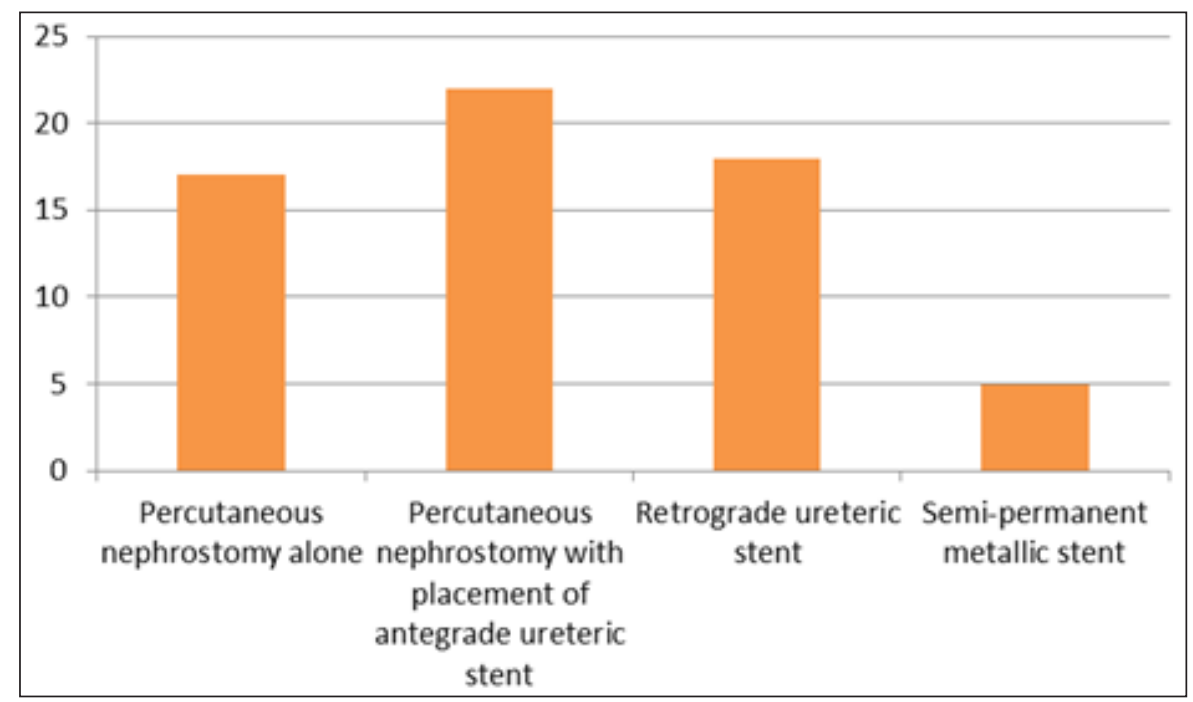

Fig. 1. Lifetime experience with various modalities for the management of extramural malignant ureteric obstruction.

neous nephrostomy tube and patients with ureteric stents is similar, significant patient preference can exist when given the choice between ureteric stents and percutaneous nephrostomy drainage [9].

Like patient factors, consultant urologists are often influenced by variables such as the availability of interventional radiology in their institution and the requests of the primary team treating the patient. The absence of guidelines in the management of extramural malignant ureteric obstruction leads to confusion in decision making and in the interaction between urology and other clinical disciplines such as medical oncology, general surgery and gynecology $[8,9]$. The aim of this study was to survey consultant urologists regarding their management of malignant extramural ureteric obstruction with the goal of achieving a better consensus regarding treatment options.

\section{Methods}

A voluntary anonymous electronic survey was sent to all consultant urologists practicing in the Republic of Ireland via email. E-mail addresses were obtained directly from the Irish Society of Urology directory. A 10-question survey was compiled and disseminated via the survey program SurveyMonkey (http://surveymonkey.com). Basic statistics were calculated by the SurveyMonkey program. Ethical approval was not required for this study. The 10-question survey are as follows:

1. In patients with extramural malignant ureteric obstruction, do you use any of the following in your practice? (tick all appropriate) o Percutaneous nephrostomy alone

o Percutaneous nephrostomy with placement of antegrade ureteral stent

o Retrograde ureteral stent

o Memokath

2. Does the presence of bilateral malignant ureteric obstruction alter your choice of tube/stent placement?

o $\mathrm{Yes} / \mathrm{No} / \mathrm{Unsure}$

o If so, how?

3. Do you preferentially stent antegrade or retrograde initially for malignant obstruction? (please tick one)

o Antegrade

o Retrograde

o Unsure

4. If a stent is initially placed antegradely, when do you arrange for the first stent change? (please tick one)

o $<2$ months

o 2-4 months

o 4-6 months

o 6-10 months

o > 10 months

o Never

o $\mathrm{n} / \mathrm{a}$

5. If a retrograde ureteral stent is initially placed, when do you arrange for the first stent change? (please tick one)

o $<2$ months

o 2-4 months

o 4-6 months

o 6-10 months

o > 10 months

o Never

o $\mathrm{n} / \mathrm{a}$

6. Does the duration of expected survival influence your decision/agreement to stent?

o $\mathrm{Yes} / \mathrm{No} / \mathrm{Unsure}$ 


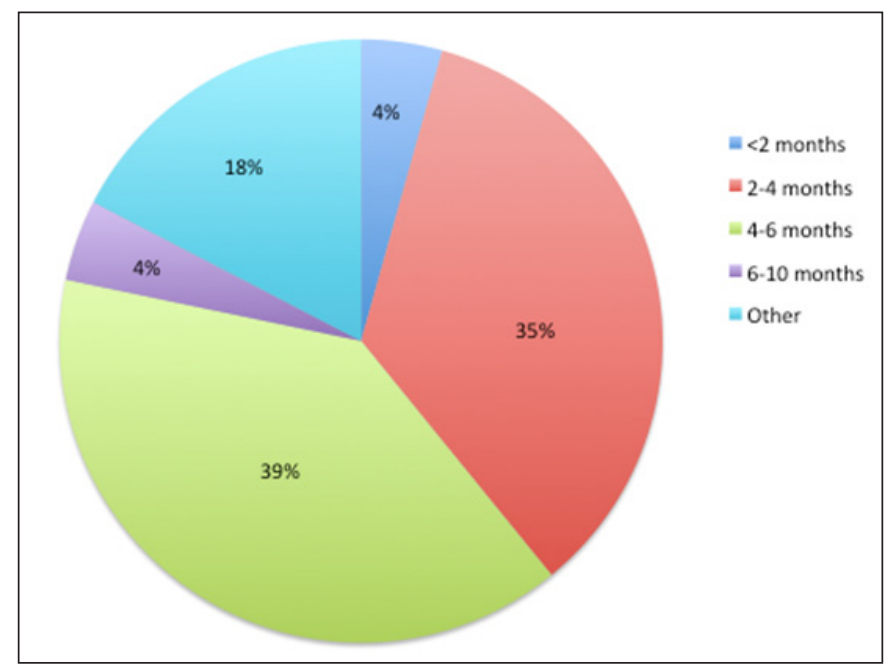

Fig. 2. Time to the first ureteric stent change after initial antegrade placement.

If so, would this survival need to be:

o $>3$ months

o $>6$ months

o $>1$ year

7. Do you or the service managing the patient re-image the patient to assess response to oncological treatment (if any)?

o Yes/No/Unsure/Not applicable

8. If you answered yes to Q7 above, would an apparent improvement in the extramural obstruction on re-imaging lead you to discontinue stenting?

o Yes/No/Maybe

9. Are you generally happy with the level of ongoing communication between urology and the primary service managing the patient?

o $\mathrm{Yes} / \mathrm{No} / \mathrm{Un}$ sure

10. Are you aware of any guidelines regarding use of ureteral stents in malignant ureteric obstruction?

o Yes/No.

o If so, please specify

\section{Results}

Responses were received from 23 of the 40 consultant urologists contacted (57.5\% response rate). Figure 1 demonstrates which treatment modalities consultants reported using in their lifetime experience for management of extramural malignant ureteric obstruction. Total 95.7\% ( $\mathrm{n}=22$ ) of consultants reported using a percutaneous nephrostomy with subsequent placement of antegrade ureteric stent in their practice while $73.9 \%(\mathrm{n}=$ 17) would use a percutaneous nephrostomy alone, $78.3 \%$

Management of Extramural Malignant Ureteric Obstruction

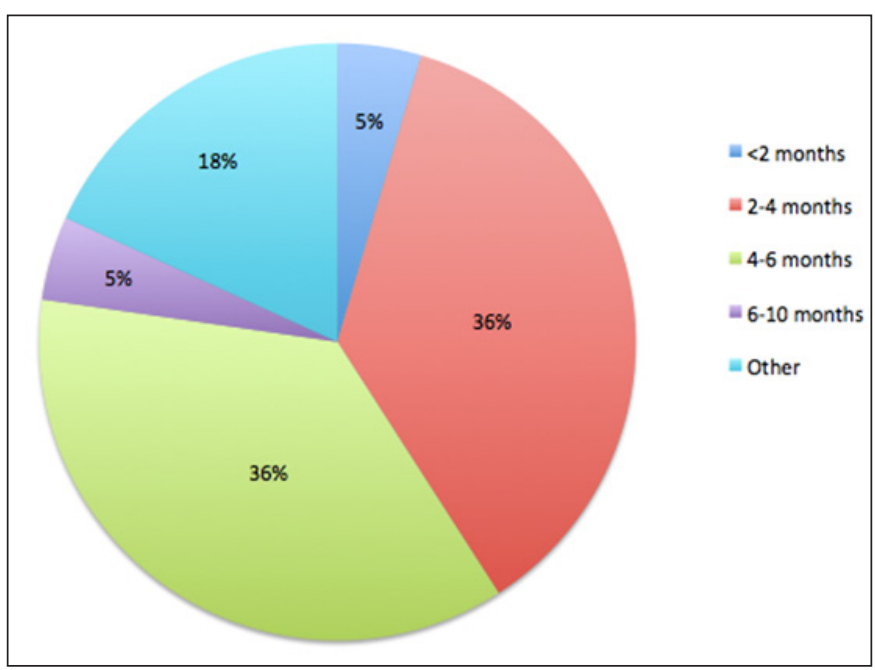

Fig. 3. Time to the first ureteric stent change after initial retrograde placement.

$(\mathrm{n}=18)$ a stent placed retrogradely and $21.7 \%(\mathrm{n}=5)$ a semi-permanent metallic stent. The majority of respondents $(82.6 \%, \mathrm{n}=19)$ did not feel that the presence of bilateral obstruction would alter their choice of tube/stent placement.

Eleven $(47.8 \%)$ respondents favor stenting retrogradely in the first instance with an equal proportion choosing an antegrade method by interventional radiology. Figure 1 and 2 demonstrate when respondents would arrange for the first stent change, depending on whether the stent was initially placed antegradely (fig. 2) or retrogradely (fig. 3 ).

The majority $(\mathrm{n}=13,59.1 \%)$ of consultant urologists replied that the life expectancy of the patient influenced their decision and agreement to place a ureteric stent, while $31.8 \%(\mathrm{n}=7)$ answered that they were unsure whether it would or not. Only 1 respondent $(4.6 \%)$ replying that expected patient survival did not impact their decision to stent. Of those respondents who felt that a patient's predicted survival influenced their decision or agreement to stent, $26.3 \%(n=5)$ felt this survival would need to be $>3$ months, with $42.1 \%(\mathrm{n}=8)$ needing it to be $>6$ months and $5.3 \%(n=1)>1$ year. Five $(26.3 \%)$ respondents felt it would depend on certain factors including patient and family wishes and suitability for a general anesthetic.

Half of consultant urologists questioned ( $\mathrm{n}=11$, $50.0 \%$ ) replied that they or the service managing the patient would re-image the patient to assess response to on- 
cological treatment (if any) with $36.4 \%(\mathrm{n}=8)$ reporting that they sometimes would. Sixteen respondents $(80.0 \%)$ felt that an apparent improvement in the extramural obstruction on repeat imaging may lead them to discontinue stenting while $15.0 \%(\mathrm{n}=3)$ said that it definitely would. The majority of respondents $(\mathrm{n}=18,81.8 \%)$ were generally happy with the level of ongoing communication between urology and the primary service managing the patient.

\section{Discussion}

This study provides valuable insights into the views and management practices of consultant urologists practicing in Ireland with regard to treatment of extramural malignant ureteric obstruction. This survey highlights that most consultant urologists would use percutaneous nephrostomy alone ( $\mathrm{n}=17,73.9 \%)$ with or without antegrade stent placement $(\mathrm{n}=22,95.7 \%)$ or retrograde stent placement $(\mathrm{n}=18,78.3 \%)$ in their practice. Only one quarter of urologists had used Memokath ${ }^{\circledR}$ stents in their practice. This most likely reflects their relatively low success rates with 1 study reporting patency rates of $51.2 \%$ with a median 15 month follow-up in patients treated for malignant ureteric obstruction [10]. The varying responses of consultants in our cohort highlight the clinical challenge in dealing with this issue. The lack of consensus guidelines is likely due to the diversity in this condition and urologists make their decision on a case by case situation.

With regard to the first stent insertion for extramural malignant ureteric obstruction, an equal number of urologists in our survey choose antegrade as retrograde stenting. In our experience, there are extrinsic logistical factors such as access to theatre and the availability of interventional radiology that influence this decision making process in addition to patient factors. A survey by Lynch et al. [11] examined the attitudes of radiologists and endourologists regarding the most effective method of urinary diversion when renal unobstruction is indicated. This demonstrated that urologists favored percutaneous nephrostomy placement more often than radiologists (69 vs. $48 \%$ ) across a range of indications including ureteric obstruction with hydronephrosis with advanced malignancy for palliation. In any instance, antegrade stenting can often be attempted if retrograde attempts fail [1]. However, these patients are often quite unwell and general anesthesia is often considered high risk. Uthappa et al. [12] attempted retrograde stent placement in a consecutive series of 50 ureters in 30 patients with malignant ureteric obstruction. The success rate of retrograde ureteric stent placement was $50 \%$ with subsequent antegrade stent placement possible in all but 1 of the remaining ureters.

The timing of the first stent change remains a contentious issue with wide variability in responses. This also likely reflects the variety in performance status, type of malignancy and location of obstruction. Jeong et al. [13] demonstrated that $16 \%(12 / 73)$ of patients who had a successful initial stent insertion went on to develop stent failure with a median (range) interval of 3.2 (1 day to 9 months).

Over half ( $\mathrm{n}=13,59.09 \%)$ of consultant urologists surveyed felt that the duration of expected patient survival influences their decision or agreement to stent. For all respondents, $42.1 \%$ felt that the projected survival would need to be greater than 6 months with 5 respondents reporting that it would be dependent on patient factors such as their wishes and suitability for a general anesthetic. It is advisable that this expected survival is discussed when allowing patients to make an informed decision with regard to the optimal management, if any, of their ureteric obstruction. Jeong et al. [13] retrospectively reviewed 86 patients who had a non-urological malignant ureteric obstruction treated by ureteric stenting and demonstrated that $48 \%$ of patients died within 1 year.

While the majority $(n=18,81.8 \%)$ of consultant urologists surveyed were generally happy with the level of ongoing communication between urology and the primary service managing the patient, it is important to be cognisant that urologists and medical oncologists often have differing opinions as to the optimal management of these patients. Hyams et al. [14] utilized a web-based survey to compare intervention preferences for extrinsic malignant ureteric obstruction. While only $15 \%$ of urologists and $12.4 \%$ of medical oncologists responded, there was significant discrepancy between their approaches to the management of hypothetical clinical scenarios. As an example, medical oncologists were more likely to recommend percutaneous nephrostomy alone as the next step after stent failure in unilateral ureteric obstruction (79 vs. $62 \%$ ), whereas urologists were more likely to suggest stent manipulation including stent exchange, upsizing, internalising, and etc ( 37 vs. $17 \%$ ). It is important to have a 3-way shared decision making process in these cases involving the appropriately informed patient, the primary physician and the urologist.

There are a number of limitations to our study. These include the fact that a number of variables such as type of malignancy and level of obstruction are not accounted 
for. We could also have further elucidated consultant preferences with regard to techniques such as placing 2 stents side by side and use of metallic stents. However, the aim of this study was to gain an overview of consultant attitudes and current practice patterns. Our response rate was in line with expected rates for online surveys and gives a good insight into national practice.

\section{Conclusion}

There is a lack of consensus regarding the management of this challenging problem, particularly with regard to timing of the first stent change and whether to use an antegrade or retrograde approach in the first instance. This reflects the heterogeneous patient cohort and the important factors of life expectancy and patient co-morbidities.

\section{References}

1 Kouba E, Wallen EM, Pruthi RS: Management of ureteral obstruction due to advanced malignancy: optimizing therapeutic and palliative outcomes. J Urol 2008;180:444-450.

2 Hsu L, Li H, Pucheril D, Hansen M, Littleton R, Peabody J, Sammon J: Use of percutaneous nephrostomy and ureteral stenting in management of ureteral obstruction. World J Nephrol 2016;5:172-181.

3 Izumi K, Mizokami A, Maeda Y, Koh E, Namiki M: Current outcome of patients with ureteral stents for the management of malignant ureteral obstruction. J Urol 2011;185: 556-561.

4 Wang JY, Zhang HL, Zhu Y, Qin XJ, Dai BO, Ye DW: Predicting the failure of retrograde ureteral stent insertion for managing malignant ureteral obstruction in outpatients. Oncol Lett 2016;11:879-883.

5 Chow PM, Hsu JS, Wang SM, Yu HJ, Pu YS, Liu KL: Metallic ureteral stents in malignant ureteral obstruction: short-term results and radiological features predicting stent failure in patients with non-urological malignancies. World J Urol 2014;32:729-736.
6 Sountoulides P, Pardalidis N, Sofikitis N: Endourologic management of malignant ureteral obstruction: indications, results, and quality -of-life issues. J Endourol 2010;24:129-142.

7 Lang EK, Winer AG, Abbey-Mensah G, Anne R, Allaei A, Friedman F, Thomas R, La Nasa J, Davis R: Long-term results of metallic stents for malignant ureteral obstruction in advanced cervical malignancy. J Endourol 2013;27:646-651.

8 Rosevear HM, Kim SP, Wenzler DL, Faerber GJ, Roberts WW, Wolf JS Jr: Retrograde ureteral stents for extrinsic ureteral obstruction: nine years' experience at University of Michigan. Urology 2007;70:846-850.

9 Joshi HB, Adams S, Obadeyi OO, Rao PN: Nephrostomy tube to 'JJ' ureteric stent in ureteric obstruction: assessment of patient perspectives using quality-of-life survey and utility analysis. Eur Urol 2001;39:695-701.

10 Shekarriz B, Shekarriz H, Upadhyay J, Banerjee M, Becker H, Pontes JE, Wood DP Jr: Outcome of palliative urinary diversion in the treatment of advanced malignancies. Cancer 1999;85:998-1003.
11 Lynch MF, Anson KM, Patel U: Current opinion amongst radiologists and urologists in the UK on percutaneous nephrostomy and ureteric stent insertion for acute renal unobstruction: results of a postal survey. BJU Int 2006;98:1143-1144.

12 Uthappa MC, Cowan NC: Retrograde or antegrade double-pigtail stent placement for malignant ureteric obstruction? Clin Radiol 2005;60:608-612.

13 Jeong IG, Han KS, Joung JY, Seo HK, Chung $\mathrm{J}$ : The outcome with ureteric stents for managing non-urological malignant ureteric obstruction. BJU Int 2007;100:1288-1291.

14 Hyams ES, Shah O: Malignant extrinsic ureteral obstruction: a survey of urologists and medical oncologists regarding treatment patterns and preferences. Urology 2008;72:5156. 